\title{
The Impact of Flash Glucose Monitoring on Markers of Glycaemic Control and Patient Satisfaction in Type 2 Diabetes
}

\author{
Ayman Al Hayek ${ }^{1}$, Mohamed Al Dawish ${ }^{1}$, Manal El Jammal ${ }^{2}$ \\ 1. Department of Endocrinology and Diabetes, Prince Sultan Military Medical City, Riyadh, SAU 2. Scientific Affairs, \\ Abbott Diabetes Care, Dubai, ARE
}

Corresponding author: Ayman Al Hayek, ayman.alhayek@yahoo.com

\begin{abstract}
Introduction: The effect of flash glucose monitoring on glycaemic control and patient satisfaction in insulin-treated type 2 diabetes (T2D) from Saudi Arabia is uncertain. The aim of this prospective observational study was to evaluate the change in HbA1c (Hemoglobin A1c) and satisfaction with treatment following the initiation of flash glucose monitoring.
\end{abstract}

Methods: This single-arm, single-centre prospective observational study included flash glucose monitoringnaive adult patients with T2D managed with multiple daily injections of insulin therapy (MDI) and HbA1c $\geqslant 7 \%$. HbA1c was measured, and the Diabetes Treatment Satisfaction Questionnaire (DTSQ, Arabic version) and Glucose Monitoring Satisfaction Survey (GMSS) were completed at baseline and 12 weeks.

Results: For participants ( $\mathrm{n}=54$ ) from one diabetes centre, HbA1c significantly improved by $0.44 \%$ from $8.22 \% \pm 0.69$ (mean \pm SD) at baseline to $7.78 \% \pm 0.71$ at 12 weeks, $\mathrm{p}<0.001$. Confirmed hypoglycaemic episodes reduced from $4.43 \pm 1.51$ episodes/month to $1.24 \pm 1.15$ ( -3.19 , $\mathrm{p}<0.001)$. Glucose monitoring frequency improved, indicated by the number of scans per day, with a mean increase of $5.13(\mathrm{p}<0.001)$ tests/day. GMSS scores improved across all four categories, as did overall treatment satisfaction ( $\mathrm{p}<0.001$ for all categories). Patients perceived clear improvements across all questions relating to satisfaction and frequency of hypo- or hyperglycaemic episodes.

Conclusion: Following initiation of flash glucose monitoring in patients with T2D and MDI insulin therapy, HbA1c improved with reduced hypoglycaemic events and increased patient-reported satisfaction. This study contributes valuable data on the use of flash glucose monitoring in this population, and a larger multicentre study is warranted to inform future health policy for T2D in Saudi Arabia.

Review began 05/24/2021 Review ended 06/16/2021 Published 06/28/2021

(๑) Copyright 2021 Al Hayek et al. This is an open access article distributed under the terms of the Creative Commons Attribution License CC-BY 4.0., which permits unrestricted use, distribution, and reproduction in any medium, provided the original author and source are credited.
Categories: Endocrinology/Diabetes/Metabolism

Keywords: diabetes mellitus, flash glucose monitoring, freestyle libre ${ }^{\circledR}$, glycaemic control, intermittent scanning continuous glucose monitoring, treatment satisfaction, type 2 diabetes

\section{Introduction}

Type 2 diabetes mellitus (T2D) is a growing public health problem globally, posing a significant challenge to healthcare systems. The onus of management rests largely upon the individual patient, and this can present a significant burden. Self-management encompasses broad factors such as diabetes medication adherence, diet, physical activity, and blood glucose self-monitoring in achieving optimal glycaemic control. Physical, social, and mental distress are well characterised phenomena in patients with diabetes, and these can impact the ability to self-manage. A number of tools are available to make various aspects of diabetes management easier for patients and hence to improve health and psychosocial outcomes. One such tool is continuous glucose monitoring (CGM), which has grown in popularity in recent years, overcoming some of the inconveniences of traditional finger-prick methods. The FreeStyle Libre ${ }^{\circledR}$ flash glucose monitoring system (Abbott Laboratories, Chicago, IL, USA) measures interstitial glucose levels via a small sensor applied to the back of the upper arm and has been described in detail elsewhere [1]. On scanning the sensor, discrete real-time glucose readings are provided. A number of studies on the use of flash glucose monitoring in T2D have emerged globally, supporting the notion that the system contributes to improvements in selfmanagement in terms of both glycaemic control and quality of life [2-8]. However, the use of flash glucose monitoring remains poorly characterised in patients with T2D in the Arab region and in Saudi Arabia in particular. Saudi Arabia was placed seventh in global rankings of T2D prevalence and second within the Middle East in a 2005 report by the World Health Organization [9]. A more recent report from the International Diabetes Federation places the Middle East and North Africa region first in regional rankings of world-age standardised diabetes prevalence [10]. This continued upward trend in the pervasiveness of T2D is accompanied by evidence of poor glycaemic control in this patient group [11, 12], which has sparked calls for more comprehensive nationwide research to enable tailoring of self-management programs to meet the needs of the T2D population in Saudi Arabia [13, 14]. In order to begin the process of addressing this data paucity, the primary aim of this prospective observational study was to introduce flash glucose monitoring 
to patients with T2D managed with multiple daily injections of insulin therapy (MDI), currently using selfmonitoring of blood glucose (SMBG), and evaluate the impact on HbA1c, treatment satisfaction, and glucose monitoring satisfaction.

\section{Materials And Methods Study design}

This prospective, observational, single-arm study was conducted over 12-weeks in a single diabetes centre in Riyadh, Saudi Arabia. Consecutive patients were invited to participate if they were; aged 20-75 years, had a diagnosis of T2D, an $\mathrm{HbA} 1 \mathrm{c} \geqslant 7 \%$ (or fasting blood glucose $\geqslant 110 \mathrm{mg} / \mathrm{dL},<250 \mathrm{mg} / \mathrm{dL}$ ), prescribed an MDI insulin regimen for at least one year, used conventional SMBG to test their glucose levels at least two times per day, had no prior experience of flash glucose monitoring or any other CGM system, and attended regular follow-up appointments at our centre. Individuals were not included if they had; a history of pancreatitis, severe infections, severe mental illnesses, or malignant disease; history of serious vascular diseases (such as stroke or myocardial infarction) within six months prior to the initiation of the study, pregnancy, or planned future pregnancy or were deemed as unfit to participate by the primary physician.

Patients attended the diabetes treatment centre for their baseline and follow-up visits as per the study protocol. Additional appointments were arranged as per the centre protocol for follow-up with the treating physician and educator.

At the baseline visit, the FreeStyle Libre sensor was attached to the back of the patient's upper arm by a trained diabetes educator. Patients were educated and trained on the proper application of the sensor and followed instructions as per the label. Patients were advised that, as far as possible, current glucose levels should be checked by scanning the sensor at least every eight hours. In addition, participants were advised to confirm sensor glucose levels with an SMBG test during unsteady glucose states, during impending or suspected hypoglycaemia, and if their sensor readings did not match their symptoms. Demonstrations were given on how sensor glucose levels could be confirmed with a capillary measurement using the blood glucose meter in-built in the reader of the flash glucose monitoring system. No additional counselling or education was provided. However, all participants were given the contact details of the diabetes educator, who could be contacted at any time during the study. At the end of the study, sensor data were downloaded to a computer to produce the reports, including the ambulatory glucose profile, in order to identify the number of scans performed during the study period.

At the baseline visit, demographic characteristics were recorded (age, sex, height and weight, and duration of diabetes) at baseline and the 12-week visits clinical characteristics (frequency of SMBG or flash glucose monitoring scans per day, number of SMBG confirmed hypoglycaemic episodes per month, the total daily dose of insulin and $\mathrm{HbA1c}$ ) were recorded. HbA1c was measured using the COBAS INTEGRA 400 plus/800 analyzers at the central laboratory of the Prince Sultan Military Medical City (PSMMC). Baseline clinical data for frequency of SMBG for the preceding four weeks and frequency of hypoglycaemic events were collected using the FreeStyle Optium Neo ${ }^{\circledR}$ blood glucose meter (Abbott Laboratories, Chicago, IL, USA) and FreeStyle Auto-Assist $\mathrm{Neo}^{\circledR}$ software.

Participants completed the Arabic versions of the Diabetes Treatment Satisfaction Questionnaire - Status Version (DTSQs) at baseline and at 12 weeks the Diabetes Treatment Satisfaction Questionnaire - Change Version (DTSQc, [15-17]). Participants also completed the Glucose Monitoring Satisfaction Survey (GMSS) version T2D at the baseline and study end visits (12 weeks). This questionnaire is focused particularly on satisfaction with the mode of glucose monitoring. Responses give rise to four summary scores: emotional burden, behavioural burden, openness, and worthwhileness, where higher scores indicated greater perceived levels of these parameters [18].

\section{Ethics}

Participants gave written informed consent to participate in the study. The study protocol was approved by the Research Ethics Committee of the PSMMC in accordance with the Helsinki Declaration of 1964, revised in 2013 (ethical approval no. 1394-HP-01-R079). All participants could withdraw at any point without reason or prior notice.

\section{Statistical analysis}

Summarized parameters were expressed as mean \pm standard deviation (SD) and range. The statistical significance of changes between patients' paired baseline and 12-week data was determined by way of the two-tailed Student's t-test or the Wilcoxon-Mann-Whitney U test, depending on outcomes of normality testing of timepoint differences $(\boldsymbol{X}=0.05)$, and were expressed as mean \pm standard deviation and $95 \%$ confidence interval (CI). The associations between clinical parameters such as HbA1c, frequency of daily monitoring, and rate of hypoglycaemic episodes were assessed by visual inspection of scatter plots and calculation of Pearson's product-moment correlation coefficient. The association between changes in clinical parameters and treatment satisfaction was calculated in a similar fashion. Additional exploratory analysis was conducted, particularly on the association of age and scan frequency. All statistical analysis 


\section{Cureus}

was conducted in $\mathrm{R}$ version 4.0.1.

\section{Outcomes}

The primary outcomes were changes in HbA1c levels, patient-reported diabetes treatment satisfaction, and patient-reported glucose monitoring satisfaction from baseline to 12 weeks. Secondary outcomes included the change in the number of SMBG confirmed hypoglycaemic episodes per month, change in body mass index (BMI), change in the weight-to-height ratio $(\mathrm{WtHr})$, and change in frequency of glucose monitoring per day.

\section{Results}

All subjects $(n=54)$ were recruited from the Diabetes Treatment Center, Prince Sultan Military Medical City, Riyadh, Saudi Arabia, between October 2019 and May 2020. Participant demographics and baseline characteristics are shown in Table 1.

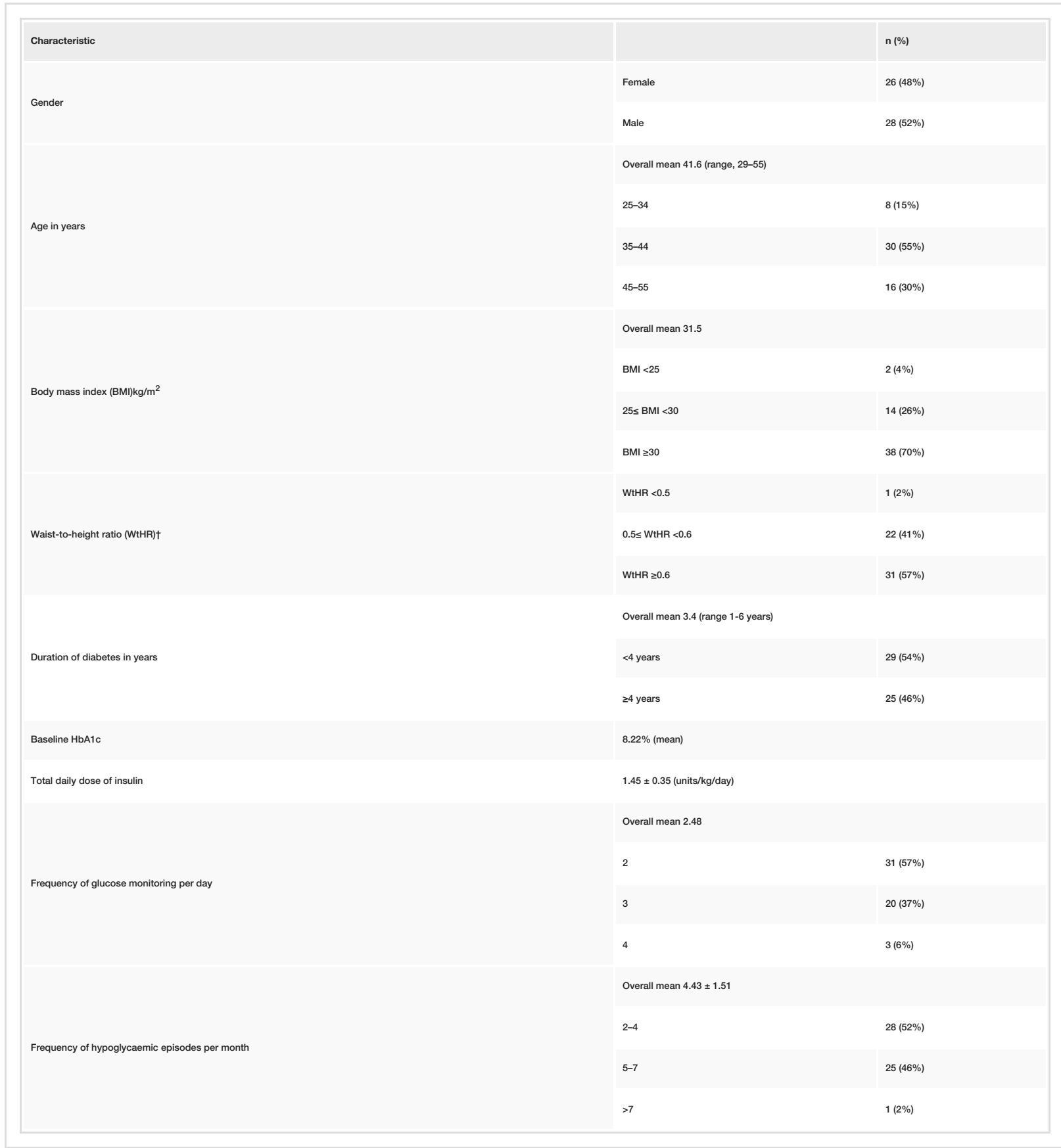

TABLE 1: Demographics and baseline characteristics of the study population $(n=54)$. 


\section{Cureus}

A statistically significant improvement was observed in HbA1c at 12 weeks, which fell by $0.44 \%(\mathrm{p}<0.001)$ from $8.22 \% \pm 0.69$ (mean \pm SD) from $7.78 \pm 0.71$. There was a greater absolute drop in HbA1c in participants with a higher HbA1c at baseline. Moreover, those with higher baseline BMI $(>30)$ experienced a greater absolute drop in $\mathrm{HbA1c}$ as well as a greater drop relative to baseline HbA1c measures (BMI $>30$, absolute $\Delta \mathrm{HbA} 1 \mathrm{c}=-0.47 \%$, relative $\Delta \mathrm{HbA} 1 \mathrm{c}=-5.54 \%$; $\mathrm{BMI}<30$, absolute $\Delta \mathrm{HbA} 1 \mathrm{c}=-0.39 \%$, relative $\Delta \mathrm{HbA} 1 \mathrm{c}=-4.45)$.

At baseline the number of confirmed hypoglycaemic episodes experienced varied from two to eight per month) which fell by a mean of 3.19 episodes per month ( $<<0.001$, Table 2$)$. Total daily dose on insulin fell by 0.43 units/kg/day $(\mathrm{p}<0.001$, Table 2$)$.

Participants performed significantly more sensor scans per day, compared to SMBG frequency at baseline, with a mean increase of 5.13 monitoring episodes per day $(\mathrm{p}<0.001$, Table 2$)$

\begin{tabular}{|c|c|c|c|c|c|}
\hline Measure & Baseline mean $\pm \mathrm{SD}$ (range) & 12-week mean $\pm \mathrm{SD}$ (range) & Mean difference & P-value & $95 \%$ confidence interval \\
\hline HbA1c\% (range) & $8.22 \pm 0.69(7.2-11.1)$ & $7.78 \pm 0.71(6.2-9.9)$ & -0.44 & $<0.001^{*}$ & $0.40,0.60$ \\
\hline Confirmed hypoglycaemic episodes per month (range) & $4.43 \pm 1.51(2-8)$ & $1.24 \pm 1.15(0-4)$ & -3.19 & $<0.001^{*}$ & $2.64,3.73$ \\
\hline Total daily dose of insulin Units/kg/day (rrange) & $1.45 \pm 0.35(0.9-2.1)$ & $1.02 \pm 0.26(0.6-1.7)$ & -0.43 & $<0.001^{*}$ & $0.35,0.55$ \\
\hline Frequency of glucose monitoring per day (range) & $2.48 \pm 0.60(2-4)$ & $7.61 \pm 1.73(4-12)$ & 5.13 & $<0.001^{*}$ & 4.50, 5.50 \\
\hline Body mass index $\left(\mathrm{kg} / \mathrm{m}^{2}\right)$ (range) & $31.5 \pm 3.04(2$ & $0.7 \pm 2.93(24.1-37.5)$ & -0.80 & $<0.001^{*}$ & $0.61,0.99$ \\
\hline Waist-to-height ratio (WtHr) (range) & $0.61 \pm 0.06(0.49-0.73)$ & $0.60 \pm 0.05(0.49-0.73)$ & -0.01 & $<0.001^{*}$ & $0.01,0.02$ \\
\hline
\end{tabular}

TABLE 2: Outcome of paired, two-tailed statistical testing of baseline against 12-week clinical parameters, where $\left(^{*}\right)$ indicates $P$-values exceeding the prespecified $\square$ of 0.05 . (SD: standard deviation.)

In correlation analysis, linear relationships were found between $\mathrm{HbA1c}$ level and daily scan frequency $(\mathrm{r}=$ $0.43, \mathrm{p}<0.001)$, between the frequency of hypoglycaemic episodes per month and daily scan frequency $(\mathrm{r}=-$ $0.68, \mathrm{p}<0.001)$, and between $\mathrm{HbA} 1 \mathrm{c}$ level and the frequency of hypoglycaemic episodes per month $(\mathrm{r}=0.32$, $\mathrm{p}<0.001$, Figure 1).
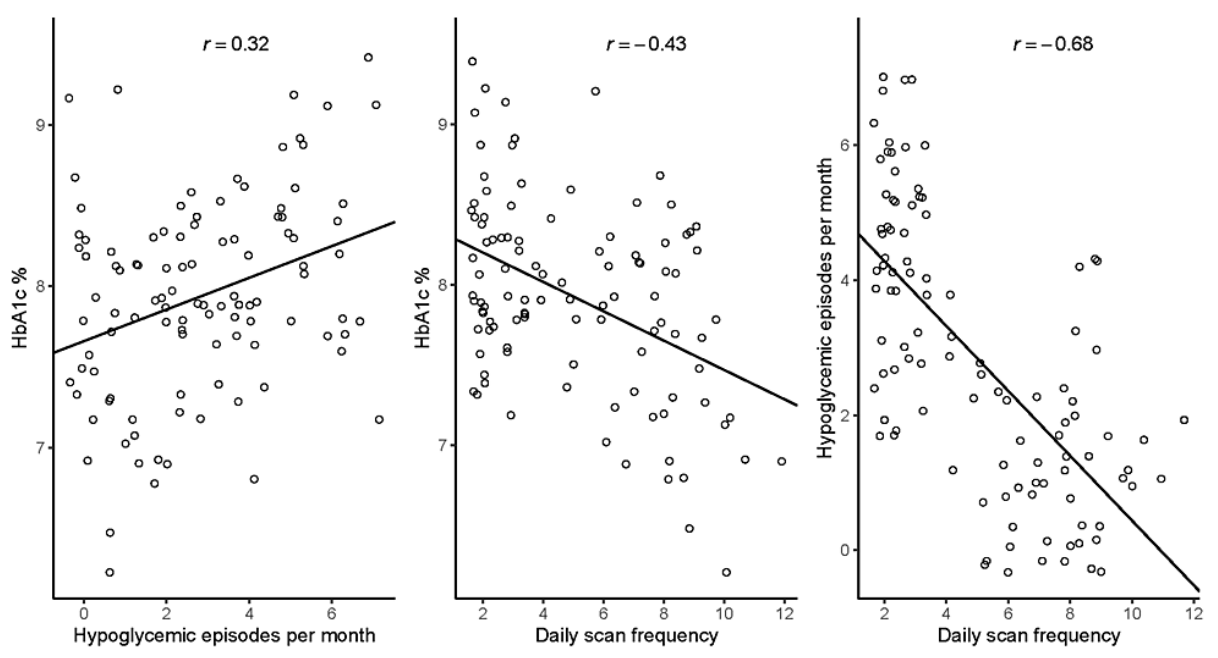

FIGURE 1: Linear relationships between glycated haemoglobin (HbA1c) level, frequency of hypoglycaemic episodes per month and daily scan frequency, over the course of the study. Lines of best fit were calculated by least squares method. Pearson's product-moment correlation coefficients are indicated by $r$.

Exploratory analysis revealed that mean scan frequency did not significantly differ when we split the cohort 


\section{Cureus}

into three age bands (25-34, 35-44, and >45 years), either at baseline or at 12 weeks.

The majority of participants experienced weight loss. The overall change in mean BMI was modest $(\mathrm{p}<0.001$, Table 2).

\section{Patient-reported satisfaction questionnaires}

Outcomes of DTSQs completed by all subjects at baseline, expressed as mean \pm standard deviation, are summarised as follows. The combined total treatment satisfaction score was $14.1 \pm 2.56$ (range, 9-20; a range of possible scores, 0 -36). The combined score of perceived episodes of hypo- or hyperglycaemia was $7.33 \pm$ 1.52 (range, 4-10; a range of possible scores, 0-12). Outcomes of DTSQc questionnaires are summarised in Figure 2, with clear perceived improvements across all questions relating to satisfaction relative to baseline. Outcomes of DTSQc also clearly evidence a reduction in the perceived rates of hypo- or hyperglycaemic episodes.
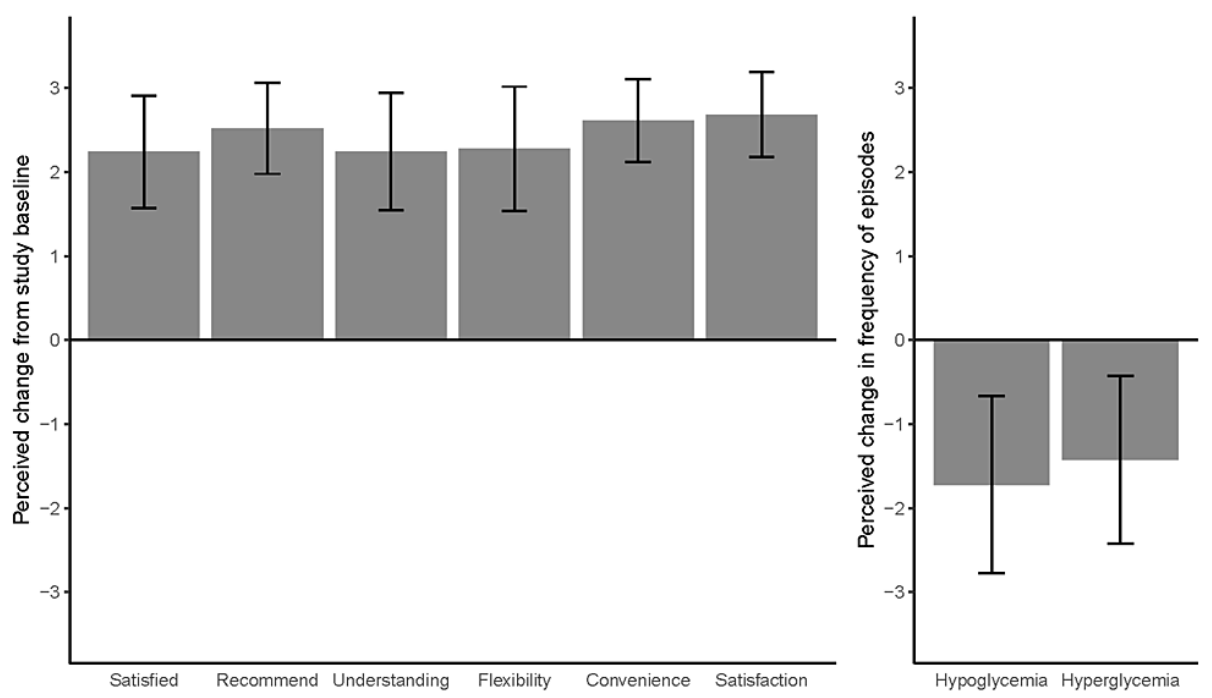

FIGURE 2: Results of the diabetes treatment satisfaction questionnaire - change version (DTSQc), expressed as mean change from study baseline \pm standard deviation (error bars).

Outcomes of the GMSS are given in Figure 3. Two-fold, statistically significant improvements were experienced from baseline to 12 weeks across all four categories of behavioural burden (14.5 \pm 2.23 vs. $5.72 \pm$ 1.19 , p <0.001, CI: 8.00, 9.50), emotional burden $(15.1 \pm 1.88$ vs. $6.87 \pm 1.58$, p <0.001, CI: 7.50, 9.00), openness $(7.59 \pm 1.65$ vs. $17.7 \pm 1.37, \mathrm{p}<0.001$, CI: $9.50,11.0)$ and worthwhileness $(6.17 \pm 1.87$ vs. $13.8 \pm 0.91$, $\mathrm{p}<0.001$, CI: 7.50, 8.00). Similarly, the total treatment satisfaction score doubled over the course of the study (32.2 \pm 4.39 vs. $66.9 \pm 2.63$, p $<0.001$, CI: $33.5,36.0)$. A negative linear correlation was identified between HbA1c levels and GMSS total treatment satisfaction $(r=-0.32, p<0.001)$, and similar correlation trends were found between HbA1c levels and the four summary categories of the GMSS. 


\section{Cureus}

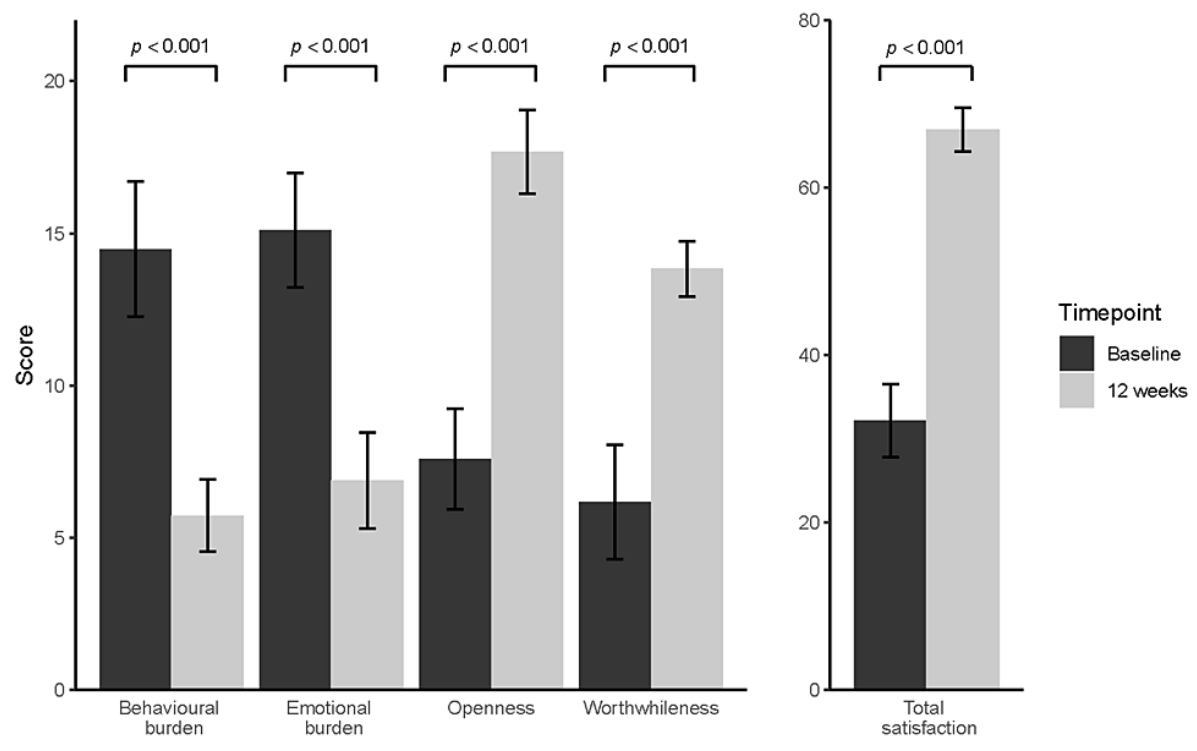

FIGURE 3: Outcomes of glucose monitoring satisfaction survey (GMSS) at baseline and 12 weeks, expressed as mean aggregated score \pm standard deviation (error bars).

\section{Discussion}

In this study, we introduced flash glucose monitoring to a cohort of patients with T2D treated with MDI therapy who had previously only used a traditional finger-prick method of blood glucose monitoring. We have shown that the introduction of flash glucose monitoring was associated with improvements in markers of glycaemic control and self-reported patient satisfaction, as well as decreases in perceived and actual episodes of hypoglycaemia, the total daily dose of insulin, BMI, and WtHr, despite a relatively short study duration. As far as we are aware, this represents first-time data on the use of flash glucose monitoring in the Saudi Arabian T2D population. Similar studies conducted in Saudi Arabia to date are limited to type 1 diabetes $[19,20]$. While these support our present findings on the positive effects of switching to flash glucose monitoring, T2D is of course a distinct entity with unique aetiologies and thus demands its own body of evidence that is apposite to Saudi Arabia. Our findings are supported by a number of observational studies, registries, and a limited number of RCTs (Randomized controlled trial) conducted elsewhere [2-8]. The REPLACE trial found significant HbA1c reductions in a pre-specified subgroup of patients under 65 years of age over a six-month period, and the switch to flash glucose monitoring resulted in a significant reduction in time spent in hypoglycaemia and significantly fewer hypoglycaemic episodes per day [3]. More recently, a reduction in HbA1c was demonstrated in a similar population of T2D managed with MDI therapy in a longer RCT from Israel and in three European chart review studies [3, 4]. In a recent meta-analysis of the safety and efficacy of flash glucose monitoring by Castellana et al (2020), which included RCTs as well as prospective and retrospective cohort studies of both type 1 diabetes and T2D, the technology was deemed an effective strategy in diabetes management [21]. Furthermore, in a real-world European analysis of over 60 million glucose tests, Dunn et al (2018) identified reductions in estimated A1c with the use of flash glucose monitoring [5]. While it has been reported that for each percentage increase in mean initial HbA1c, (for example above 7\%), the expected mean change in final HbA1c falls by $0.31 \%$ [22], the observed reduction in $\mathrm{HbA1c}$ should be interpreted cautiously as a similar reduction was reported for the control arm of an RCT in the same population [3]. Similarly, the identified trends between BMI and HbA1c change are hypothesisgenerating and remain to be explored further. The need to understand which clinical factors represent significant drivers of HbA1c reduction in the broader Saudi population is ever-present and could be explored in a larger randomised controlled trial. The reason for the observed improvement in glycaemic control in the present study is unclear. The mean total daily dose of insulin decreased from baseline to study end and most subjects lost a modest amount of weight, with differences in the paired analysis found to be statistically significantly different from baseline to 12 weeks. Speculatively, these changes may be a consequence of clinical review and participants' lifestyle modifications, either independently or in combination. In particular, weight loss is potentially a positive benefit from decreased insulin doses. However, during the study, these activities were supported by more comprehensive flash glucose data which suggests that this was a factor and this rationale is supported by recent studies [2-4]. Furthermore, hypoglycaemic episodes decreased from baseline to study end which also makes the use of comprehensive glucose data to support insulin dose requirements and titration at specific times of day more likely.

These observations of improved HbA1c with a reduction in hypoglycaemic episodes are notable in a 
population with T2D managed with MDI therapy. Furthermore, the modest change in BMI is clinically important and highly pertinent. The prevalence of obesity in the Saudi population is a major concern as it has continued to increase and it is estimated that up to 7 out of every 10 people could be classified as obese $[23,24]$. In the current study, the vast majority of participants were overweight or obese, with a WtHr in the suggestive of risk or high risk (>0.6) categories (Table 1, [25]). Recently reported data suggests there is a strong association between WHtR and cardiac risk in individuals from Saudi Arabia. As it is established that T2D already carries an increased risk for cardiovascular morbidity and mortality [26], the potential of weight loss in a population with T2D deserves further investigation in a future study [27].

In the present study cohort, reductions in HbA1c were experienced fairly systematically by all subjects. Those with higher baseline HbA1c experienced greater reductions in HbA1c, both absolutely and as a proportion of baseline measures, which has been reported by others [3, 4]. HbA1c reduction was associated with a reduction in episodes of hypoglycaemia, as well as an increase in the frequency of glucose monitoring with the number of scans carried out by patients. This observation supports real-world data in European populations [5]. Daily glucose monitoring at baseline was low compared to a European cohort [2] and similar to the previously reported frequency of glucose monitoring for Saudi Arabia [28]. Monitoring frequency increased with the use of flash glucose monitoring in this study population with MDI therapy which has been observed previously in Europe [2] and in type 1 diabetes in Saudi Arabia [19, 20]. Also encouraging is the finding that flash glucose monitoring scan frequency in the current study did not vary by age group, which supports the notion that this technology device is easy to use.

From our present study, which included measurements of patient-reported satisfaction, we surmise that convenient access to their interstitial glucose measures might be empowering for patients. The observed scanning rate which was three times that of SMBG frequency at baseline would seem to support this rationale. A recent meta-analysis by Cowart et al (2020) of RCTs studying the impact of flash glucose monitoring in both type 1 diabetes and T2D identified greater patient satisfaction and lower diabetes distress with flash glucose monitoring as compared with usual care [29]. Our study identified correlations between measures of patient satisfaction and clinical measures, which serve as cross-validity as well as underscoring the central importance of patients' wellbeing with regard to adherence to treatment regimens. This is particularly pertinent in diabetes, where issues of societal stigma are still prevalent and pervasive, accentuated by the fact that the burden of management rests upon the patients themselves.

This study was subject to a number of limitations that should be acknowledged. The cohort of participants was small and the study was conducted within a single diabetes centre. As such these results may not reflect possible outcomes at different centres or regions of Saudi Arabia. However, the baseline HbA1c and mean age of participants is similar to that of the large Middle Eastern cohort in the Global HAT study [30] which may support the generalisability of our findings. This was a relatively short study and it is not known if the reductions in $\mathrm{HbA1c}$, hypoglycaemic episodes, and weight loss would be maintained over a longer period. The present study provides novel evidence from standard clinical care settings in Saudi Arabia, however, the observational methodology limited recording of certain clinical parameters, such as more detailed glucose metrics and percentage time in ranges, which would have added further interest. In future studies, it would be useful to know how frequently clinical reviews took place, what medication adjustments were made, and what glycaemic changes occurred with an analysis of sensor data. It would also be valuable to understand how patients' perceptions of quality of life, as well as clinical changes, are sustained or indeed exceeded over a greater period of follow-up. Yaron et al (2019) provide evidence that additional counselling throughout the study period may improve the modification of lifestyle risk factors [3].

This study experience could be valuable to contribute to the design of a clinical trial. A future longer-term study could include RCT methodology and characterisation of all glycaemic metrics and lifestyle behaviours. Such a study would provide valuable insights into individualising management strategies to meet the unique needs of the T2D MDI population in Saudi Arabia.

\section{Conclusions}

This single centre, 12-week study provides valuable, novel data on the use of flash glucose monitoring in patients with T2D and MDI therapy in Saudi Arabia. The findings were an improved HbA1c and reduction in hypoglycaemic episodes with increased satisfaction with treatment after switching to flash glucose monitoring. The inclusion of this glucose monitoring technology to support modern management of T2D may have the potential to ease some of the social and economic burden of diabetes management in Saudi Arabia. The finding of significant improvements in glycaemic control and patient satisfaction after switching to flash glucose monitoring would benefit from validation in a larger multi-centre study in order to inform future health policy for the growing population of patients with T2D.

\section{Additional Information \\ Disclosures}

Human subjects: Consent was obtained or waived by all participants in this study. Research Ethics Committee of the Prince Sultan Military Medical City issued approval 1394-HP-01-R079. The study protocol 
was approved by the Research Ethics Committee of the Prince Sultan Military Medical City in accordance with the Helsinki Declaration of 1964, revised in 2013 (ethical approval no. 1394-HP-01-R079). . Animal subjects: All authors have confirmed that this study did not involve animal subjects or tissue. Conflicts of interest: In compliance with the ICMJE uniform disclosure form, all authors declare the following: Payment/services info: All authors have declared that no financial support was received from any organization for the submitted work. Financial relationships: Manal El Jammal declare(s) employment from Abbott Diabetes Care . Manal El Jammal is an employee of Abbott, Diabetes Care, United Arab Emirates. Other relationships: Ayman A. Al Hayek, Mohamed A. Al Dawish have nothing to disclose. Manal El Jammal is an employee of Abbott, Diabetes Care, United Arab Emirates.

\section{Acknowledgements}

The authors thank all the study participants and site staff for their time, effort and commitment. The authors thank Rysia Burmicz from IMR Press Limited, United Kingdom, for providing medical writing assistance and Amanda Cartmale (Scientific Affairs, Abbott, Diabetes Care, Italy) for editorial assistance with manuscript preparation.

\section{References}

1. Bailey T, Bode BW, Christiansen MP, Klaff LJ, Alva S: The performance and usability of a factory-calibrated flash glucose monitoring system. Diabetes Technol Ther. 2015, 17:787-794. 10.1089/dia.2014.0378

2. Haak T, Hanaire H, Ajjan R, Hermanns N, Riveline JP, Rayman G: Flash glucose-sensing technology as a replacement for blood glucose monitoring for the management of insulin-treated type 2 diabetes: a multicenter, open-label randomized controlled trial. Diabetes Ther. 2017, 8:55-73. 10.1007/s13300-0160223-6

3. Yaron M, Roitman E, Aharon-Hananel G, et al.: Effect of flash glucose monitoring technology on glycemic control and treatment satisfaction in patients with type 2 diabetes . Diabetes Care. 42:1178-1184. 10.2337/dc18-0166

4. Kröger J, Fasching P, Hanaire H: Three European retrospective real-world cart review studies to determine the effectiveness of flash glucose monitoring on HbA1c in adults with type 2. Diabetes Ther. 2020, 11:279291. 10.1007/s13300-019-00741-9

5. Dunn TC, Xu Y, Hayter G, Ajjan RA: Real-world flash glucose monitoring patterns and associations between self-monitoring frequency and glycaemic measures: A European analysis of over 60 million glucose tests. Diabetes Res Clin Pract. 2018, 137:37-46. 10.1016/j.diabres.2017.12.015

6. Anjana RM, Kesavadev J, Neeta D, et al.: A Multicenter Real-Life Study on the Effect of Flash Glucose Monitoring on Glycemic Control in Patients with Type 1 and Type 2 Diabetes. Diabetes Technol Ther. 2017, 19:533-540. 10.1089/dia.2017.0063

7. Wada E, Onoue T, Kobayashi T, et al.: Flash glucose monitoring helps achieve better glycemic control than conventional self-monitoring of blood glucose in non-insulin-treated type 2 diabetes: a randomized controlled trial. BMJ Open Diabetes Res Care. 2020, 8:e00111. 10.1136/bmjdrc-2019-001115

8. Ida S, Kaneko R, Imataka K, et al.: Effects of flash glucose monitoring on dietary variety, physical activity, and self-care behaviors in patients with diabetes . J Diabetes Res. 2020, 2020:9463648.

10.1155/2020/9463648

9. Pedoe HT: Preventing chronic diseases. A vital investment: WHO global report . World Health Organization, Geneva; 2005. 10.1093/ije/dyl098

10. Saeedi P, Petersohn I, Salpea P, et al.: Global and regional diabetes prevalence estimates for 2019 and projections for 2030 and 2045: Results from the International Diabetes Federation Diabetes Atlas, 9th edition. Diabetes Res Clin Pract. 2019, 157:107843. 10.1016/j.diabres.2019.107843

11. Alramadan MJ, Magliano DJ, Almigbal TH, et al.: Glycaemic control for people with type 2 diabetes in Saudi Arabia - an urgent need for a review of management plan. BMC Endocr Disord. 2018, 18:62. 10.1186/s12902018-0292-9

12. Al Slamah T, Nicholl BI, Alslail FY, Harris L, Kinnear D, Melville CA: Correlates of type 2 diabetes and glycaemic control in adults in Saudi Arabia a secondary data analysis of the Saudi health interview survey. BMC Public Health. 2020, 20:515. 10.1186/s12889-020-08597-6

13. Al Dawish MA, Robert AA, Braham R, Al Hayek AA, Al Saeed A, Ahmed RA, Al Sabaan FS: Diabetes mellitus in Saudi Arabia: A review of the recent literature. Curr Diabetes Rev. 2016, 12:359-368. 10.2174/1573399811666150724095130

14. Robert AA, Al Dawish MA, Braham R, Musallam MA, Al Hayek AA, Al Kahtany NH: Type 2 Diabetes Mellitus in Saudi Arabia: Major Challenges and Possible Solutions. Curr Diabetes Rev. 2017, 13:59-64. 10.2174/1573399812666160126142605

15. Bradley C: The diabetes treatment satisfaction questionnaire: DTSQ. Handbook of psychology and diabetes: A guide to psychological measurement in diabetes research and practice. Bradley C (ed): Harwood Academic Publishers, Chur, Switzerland; 1994. 111-32.

16. Bradley C, Plowright R, Stewart J, Valentine J, Witthaus E: The diabetes treatment satisfaction questionnaire change version (DTSQc) evaluated in insulin glargine trials shows greater responsiveness to improvements than the original DTSQ. Health Qual Life Outcomes. 2007, 5:57. 10.1186/1477-7525-5-57

17. Wilbur K: Diabetes treatment satisfaction questionnaire - an Arabic adaptation for Qatar . Diabetes Res Clin Pract. 2013, 99:e24-6. 10.1016/j.diabres.2012.11.007

18. Polonsky WH, Fisher L, Hessler D, Edelman SV: Development of a new measure for assessing glucose monitoring device-related treatment satisfaction and quality of life. Diabetes Technol Ther. 2015, 17:657663. 10.1089/dia.2014.0417

19. Al Hayek AA, Robert AA, Al Dawish MA: Evaluation of FreeStyle Libre flash glucose monitoring system on glycemic control, health-related quality of life, and fear of hypoglycemia in patients with type 1 diabetes. 
Clin Med Insights Endocrinol Diabetes. 2017, 10:1179551417746957. 10.1177/1179551417746957

20. Al Hayek AA, Robert AA, Al Dawish MA: Differences of FreeStyle Libre flash glucose monitoring system and finger pricks on clinical characteristics and glucose monitoring satisfactions in type 1 diabetes using insulin pump. Clin Med Insights Endocrinol Diabetes. 2019, 12:1179551419861102. 10.1177/1179551419861102

21. Castellana M, Parisi C, Di Molfetta S, et al.: Efficacy and safety of flash glucose monitoring in patients with type 1 and type 2 diabetes: a systematic review and meta-analysis. BMJ Open Diabetes Res Care. 2020, 8:10.1136/bmjdrc-2019-001092

22. Evans M, Welsh Z, Ells S, Seibold A: The impact of flash glucose monitoring on glycaemic control as measured by HbA1c: A meta-analysis of clinical trials and real-world observational studies. Diabetes Ther. 2020, 11:83-95. 10.1007/s13300-019-00720-0

23. DeNicola E, Aburizaiza OS, Siddique A, Khwaja H, Carpenter DO: Obesity and public health in the Kingdom of Saudi Arabia. Rev Environ Health. 2015, 30:191-205. 10.1515/reveh-2015-0008

24. Memish ZA, El Bcheraoui C, Tuffaha M, et al.: Obesity and associated factors--Kingdom of Saudi Arabia, 2013. Prev Chronic Dis. 2014, 11:E174. 10.5888/pcd11.140236

25. Alshamiri MQ, Mohd A Habbab F, Al-Qahtani SS, Alghalayini KA, Al-Qattan OM, El-Shaer F: Waist-toheight ratio (WHtR) in predicting coronary artery disease compared to body mass index and waist circumference in a single center from Saudi Arabia. Cardiol Res Pract. 2020, 2020:4250793. $10.1155 / 2020 / 4250793$

26. Sarwar N, Gao P, Seshasai SR, et al.: Diabetes mellitus, fasting blood glucose concentration, and risk of vascular disease: a collaborative meta-analysis of 102 prospective studies. Lancet. 2010, 375:2215-22. 10.1016/S0140-6736(10)60484-9

27. Bertoluci MC, Rocha VZ: Cardiovascular risk assessment in patients with diabetes . Diabetol Metab Syndr. 2017, 9:25. 10.1186/s13098-017-0225-1

28. Ba-Essa EM, Mobarak EI, Alghamdi A, Al-Daghri NM: Intensified glucose self-monitoring with education in Saudi DM patients. Int J Clin Exp Med. 2015, 8:19374-80.

29. Cowart K, Updike W, Bullers K: Systematic review of randomized controlled trials evaluating glycemic efficacy and patient satisfaction of intermittent-scanned continuous glucose monitoring in patients with diabetes. Diabetes Technol Ther. 2020, 22:337-45. 10.1089/dia.2019.0345

30. Khunti K, Alsifri S, Aronson R, et al.: Rates and predictors of hypoglycaemia in 27585 people from 24 countries with insulin-treated type 1 and type 2 diabetes: the global HAT study. Diabetes Obes Metab. 2016, 18:907-15. 10.1111/dom.12689 